\title{
Correlation between pain assessment scale and neurovascular compression distance to the root exit zone in trigeminal neuralgia analysis using 3D CISS MRI sequence
}

\author{
Harry Topan, ${ }^{1}$ Rahmad Mulyadi, ${ }^{1}$ Setyo Widi Nugroho, ${ }^{2}$ Joedo Prihartono ${ }^{3}$
}

pISSN: 0853-1773 • elSSN: 2252-8083 https://doi.org/10.13181/mji.v28i2.1796 Med J Indones. 2019;28:134-40

Received: October 15, 2018

Accepted: April 1, 2019

\section{Authors' affiliations:}

${ }^{1}$ Department of Radiology, Faculty of Medicine, Universitas Indonesia, Cipto Mangunkusumo Hospital, Jakarta, Indonesia, ${ }^{2}$ Department of Neurosurgery, Faculty of Medicine, Universitas Indonesia, Cipto Mangunkusumo Hospital, Jakarta, Indonesia, ${ }^{3}$ Department of Community Medicine, Faculty of Medicine, Universitas Indonesia, Jakarta, Indonesia

\section{Corresponding author:}

Rahmad Mulyadi

Department of Radiology, Faculty of Medicine, Universitas Indonesia, Cipto Mangunkusumo Hospital, Jalan Diponegoro No. 71, Kenari, Senen, Central Jakarta 10310, DKI Jakarta, Indonesia.

Tel/Fax: +62-21-3910462

E-mail: dr_rahmad_radiologi@yahoo. com

\begin{abstract}
BACKGROUND Trigeminal neuralgia (TN) is primarily caused by neurovascular compression (NVC) at root exit zone (REZ) in cerebellopontine angle cistern. In some NVC cases, it was suspected that clinical symptoms may be correlated with the distance of trigeminal nerve root to vascular contact. Pain assessment scale (PAS) was the most common scale used to evaluate TN pain, therefore this study was conducted to analyze the correlation between PAS usingnumeric rating scale (NRS) and distance from the NVC to REZ location in patients with TN using 3D CISS MRI sequence.
\end{abstract}

METHODS This cross-sectional study was conducted at the Department of Radiology, Faculty of Medicine, Universitas Indonesia, Cipto Mangunkusumo Hospital, Jakarta, using secondary data of 32 patients, collected from Picture Archiving and Communication System from January 2013 to January 2016. Statistical analysis was performed using SPSS, version 20.0. Spearman $p$-value of $<0.05$ was considered significant.

RESULTS A total of 32 patients met the inclusion criteria. The mean (SD) distances from the NVC to the REZ were 2.1 (2.1), 2.31 (2.25), and 3.22 (2.63) mm on the shortest, medial, and lateral sides, respectively. The correlation coefficients ( $r$ ) between the PAS value and the NVC distance in relation to the trigeminal nerve REZ were -0.39 ( $p=$ $0.021),-0.57(p<0.01)$, and $-0.57(p=0.294)$ on the shortest, lateral, and medial sides, respectively.

CONCLUSIONS PAS using the NRS instrument exhibited an inverse correlation to NVC distance to the REZ of the trigeminal nerve. Shorter distance increased the PAS value.

KEYWORDS neurovascular compression, pain assessment scale, trigeminal neuralgia
Trigeminal neuralgia (TN) or tic douloureux is a condition manifested by sudden unilateral facial pain along the course of one or more of the 5 th cranial nerve branches. It can occur spontaneously or be provoked by activities. ${ }^{1}$ Joffroy et $\mathrm{al}^{2}$ reported that the incidence of TN ranged from 3 to 5 cases per 100,000 people per year and the peak age of onset was 50-60 years. The TN ratio between females and males was reported as 2:1. The quality of pain in patients with TN varies. The pain is evaluated using a pain assessment scale (PAS) by a numeric rating scale (NRS), which is one of the most commonly used instruments. This diagnostic instrument is recommended by The National Initiative on Pain Control and has been used for pain evaluation before and after a procedure. ${ }^{3}$

TN is frequently caused by neurovascular compression (NVC) on the superior cerebellar artery (SCA) is most frequent vascular compressed the nerve. The other causes (about 1-2\%) include tumors in the cerebellopontine angle (CPA) region 
such as meningioma, epidermoid tumor, acoustic neuroma, vascular malformation, and multiple sclerosis. ${ }^{4}$ Neurovascular contacts caused by vessel juxtaposition in the trigeminal nerve which also occur in asymptomatic patients and neurovascular contact occurs in the proximal root exit zone (REZ) in $76 \%$ of symptomatic and $17 \%$ of asymptomatic patients. ${ }^{5}$

Suzuki et $\mathrm{al}^{6}$ reported that in asymptomatic patients with TN, the location of the NVC to the REZ was more likely to be on the distal side than that in symptomatic patients. REZ is the site where the trigeminal nerve exits the brainstem and the central nervous system transitions into the peripheral nervous system. It is in this transitional zone where NVC is likely to occur, and focal demyelination of the trigeminal nerve fibers also occurs at this site because of nerve compression by blood vessels. According to Peker et $\mathrm{al}^{7}$ the central myelin consists of oligodendroglial cells whose amount decreases as they extend more into the periphery before being replaced by Schwann cells. Demyelination in the central myelin can result in increased trigeminal pain, especially in the proximal REZ because of the higher amount of oligodendroglial cells in the proximal than in the distal REZ.

The best modality to evaluate NVC in TN is using 3D constructive interference in steady state (CISS) magnetic resonance imaging (MRI). This method has the advantages of short-time acquisition, high signal-to-noise ratio, and good contrast-to-noise ratio compared with other MRI sequences. ${ }^{8}$ Tarnaris et al ${ }^{9}$ reported that the sensitivity of $3 \mathrm{D}$ CISS MRI sequence was $100 \%$ higher than that of magnetic resonance angiography using gadolinium contrast, whose sensitivity was $56.2 \%$, when evaluating patients with hemifacial spasm. Due to the high sensitivity of 3D CISS MRI sequence in evaluating NVC, this technique can be used as a routine protocol for evaluating NVC in TN.

The distance between NVC location and REZ may be closely associated with the variation of pain quality that can be assessed by PAS as a symptom in patients with TN. To the best of found knowledge, no study in Indonesia has yet evaluated the distance between NVC location and the REZ and the vascular caliber, the vascular origin, and the grading of compression using the highly sensitive 3D CISS MRI for imaging NVC in TN. With this background, this study was conducted to analyze the correlation between PAS using NRS and the distance between NVC location and REZ in patients with TN using $3 \mathrm{D}$ CISS MRI sequence.
We expect that the results of this study would help physicians determine the appropriate therapy and in mapping for microvascular decompression and provide more information about the location of NVC to the REZ, which is a factor that can affect pain in TN.

\section{METHODS}

This retrospective, cross-sectional study was conducted using the secondary data of 32 patients, which have been collected from a picture archiving and communication system, and their PAS values collected from the medical records data from January 2013 to January 2016. The secondary data were obtained from 3D CISS MRI sequence examination by a radiographer at the Department of Radiology, Cipto Mangunkusumo Hospital. The ethical committee of the Faculty of Medicine, Universitas Indonesia, approved this study (No. 145/UN2.F1/ETIK/2016). Patients with tumor in the CPA region were excluded.

\section{Data collection}

All the study patients were imaged by MRI 1.5 T Avanto siemens. The CISS sequence parameters were repetition time (TR), $5.42 \mathrm{~ms}$, echo time (TE), $2.42 \mathrm{~ms}$, flip angle $77^{\circ}$, bandwidth $399 \mathrm{~Hz} / \mathrm{Px}$, slice thickness 2-6 mm with an interslice gap of $20 \%$, and matrix size $256 \times 256$. The region of interest was focused in the CPA to evaluate the tumor using the $\mathrm{T}_{1} \mathrm{WI}$ and $\mathrm{T}_{2} \mathrm{~W} I$ sequences in the axial, coronal, and sagittal 54 slices. The distance between the compression location and the medial and lateral REZ was measured by $3 \mathrm{D}$ CISS MRI sequence with the axial slice in the workstation monitor and the lowest distance was taken for analysis. If the vascular compression was found on one side of the trigeminal nerve, only one side measurement was done. To evaluate the vascular origin, the vascular caliber and the degree of compression were measured by $3 \mathrm{D}$ CISS MRI sequence. The distance of the compression location was defined by the distance between the vascular-nerve contact and the REZ in the anterior or lateral pons. Suzuki et al ${ }^{6}$ classified the distances as $\leq 1,1-2,2-3,3-4,4-5$, and $>5 \mathrm{~mm}$. The degree of vascular compression was obtained from 3D CISS MRI sequence examination with the axial slice for cerebrospinal fluid rim sign and deviation sign. Jo et $\mathrm{al}^{10}$ stated that the degree of vascular compression can be rated on a $0-3$ scale, with grade 0 indicating 
no vessel compression, grade 1 representing vessel compression without nerve indentation, grade 2 indicating mild-to-moderate indentation of the nerve compression, and grade 3 indicating severe indentation or displacement in the nerve by the vessel.

PAS data were obtained from the medical records of patients who underwent the 3D CISS MRI sequence examination. The numeric pain rating scale instrument was used to evaluate PAS with a range of $0-10$, where 0 implies no pain, 5 indicates moderate pain, and 10 implies worst possible pain. ${ }^{3}$

\section{Data analysis}

Statistical analysis was performed using the Statistical Package for Social Sciences, version 20.0. The correlation was measured by the Spearman test as the data were not normally distributed. The significance of the correlation was considered based on the p-value, and the strength of the correlation was determined by the $r$-value. The adjusted $r$ was obtained by the adjustment of correlation.

\section{RESULTS}

A total of 32 patients were included in the analysis. The mean (SD) of age was 53.1 (11.9) years, with the age range being 19-74 years. The proportion of females ( $n=21,65.5 \%$ ) was higher than that of males (34.4\%). The majority of subjects were diagnosed with TN on the right side (59.4\%), whereas TN on the left side was detected in 13 subjects (40.6\%). Based on the quality of TN pain that was visualized by NRS, approximately 17 subjects were found to suffer from pain on a scale of (53.1\%) (Table 1).

In 29 subjects, the source of trigeminal nerve compression was a single vascular origin, and in 3 subjects, it was two vascular origins. Thus, there were 32 subjects with a total of 35 vascular origin measurement that was caused by compression in the trigeminal nerve. The most common vascular origin involved in NVC was the SCA in both right-sided TN (52.38\%) and left-sided TN (57.14\%) (Table 2). Among 35 vascular origins that caused the NVC in patients with $\mathrm{TN}$, the largest vascular caliber was that of the basilar artery and the smallest was that of the pontine artery.

In this study, it was used the shortest distance between REZ and NVC in the trigeminal nerve for evaluating the correlation between the distance and
Table 1. Distribution of subject characteristics distance between NVC and REZ in TN

\begin{tabular}{|c|c|}
\hline Subject characteristics & $\begin{array}{c}\text { Frequency, } \\
\mathrm{n}(\%)\end{array}$ \\
\hline \multicolumn{2}{|l|}{ Gender } \\
\hline Male & $11(34.4)$ \\
\hline Female & $21(65.5)$ \\
\hline \multicolumn{2}{|l|}{ Age groups (years) } \\
\hline $19-50$ & $14(43.8)$ \\
\hline $51-74$ & $18(56.3)$ \\
\hline \multicolumn{2}{|l|}{ Side of TN diagnosis } \\
\hline Right & $19(59.4)$ \\
\hline Left & $13(40.6)$ \\
\hline \multicolumn{2}{|l|}{ Pain rating scale } \\
\hline Null & $6(18.7)$ \\
\hline $1-2$ & $9(28.1)$ \\
\hline$\geq 3$ & $17(53.1)$ \\
\hline \multicolumn{2}{|l|}{ Vascular origin } \\
\hline 1 origin & $29(91.4)$ \\
\hline 2 origins & $3(8.6)$ \\
\hline \multicolumn{2}{|l|}{ Grade of right compression scale } \\
\hline Null & $13(37.1)$ \\
\hline 1 & $9(25.7)$ \\
\hline 2 & 8 (22.9) \\
\hline 3 & $5(14.3)$ \\
\hline \multicolumn{2}{|l|}{ Grade of left compression scale } \\
\hline Null & $14(40)$ \\
\hline 1 & $5(14.3)$ \\
\hline 2 & $4(11.4)$ \\
\hline 3 & $12(34.3)$ \\
\hline \multicolumn{2}{|c|}{ Type of vascular crossing to trigeminal nerve } \\
\hline Type A & $4(12.5)$ \\
\hline Type B & 7 (21.9) \\
\hline Type C & $18(56.3)$ \\
\hline Unclassified ( 2 origins involved) & $3(9.8)$ \\
\hline \multicolumn{2}{|c|}{ Distance between NVC and REZ, mean (SD) } \\
\hline The shortest distance & $2.1(2.1)$ \\
\hline Medial side & $2.31(2.255)$ \\
\hline Lateral side & $3.22(2.637)$ \\
\hline
\end{tabular}

$\mathrm{NVC}=$ neurovascular compression; $\mathrm{REZ}=$ root exit zone; $\mathrm{TN}=$ trigeminal neuralgia; $\mathrm{SD}=$ standard deviation

the PAS value. The mean (SD) of the measurement of the shortest distance was 2.1 (2.1) $\mathrm{mm}$. Direct measurement was used to evaluate the distance between REZ and NVC in the trigeminal nerve on the medial and lateral sides. The mean (SD) of the measurement of the distance between the vascular 
Table 2. Vascular caliber according to vascular origin in TN

\begin{tabular}{|c|c|c|c|c|}
\hline \multirow[b]{2}{*}{ Vascular origin } & \multicolumn{2}{|c|}{ Right } & \multicolumn{2}{|c|}{ Left } \\
\hline & Frequency, n (\%) & $\begin{array}{l}\text { Vascular caliber, } \\
\text { mean (SD) }\end{array}$ & Frequency, n (\%) & $\begin{array}{c}\text { Vascular caliber, } \\
\text { mean (SD) }\end{array}$ \\
\hline SCA & $11(52.38)$ & $1.29(0.19)$ & $8(57.14)$ & \\
\hline AICA & $3(14.29)$ & $1.30(0.15)$ & $4(28.57)$ & \\
\hline Basilar artery & $2(9.52)$ & $7.09(1.22)$ & $1(7.14)$ & \\
\hline Vertebral artery & - & 5.35 & $1(7.14)$ & \\
\hline Pontine artery & $3(14.29)$ & $0.75(0.98)$ & - & \\
\hline Pet. superior vein & $2(9.52)$ & $0.97(0.36)$ & - & \\
\hline
\end{tabular}

$\mathrm{TN}=$ trigeminal neuralgia; $\mathrm{SD}=$ standard deviation; $\mathrm{SCA}=$ superior cerebellar artery; $\mathrm{AICA}=$ anteroinferior cerebellar artery; Pet. superior vein=superior petrous vein

compression and the REZ on the medial side was $2.31(2.255) \mathrm{mm}$, and the shortest distance was approximately $0 \mathrm{~mm}$, whereas the longest distance was approximately $10.00 \mathrm{~mm}$. On the lateral side, we found that the mean (SD) was $3.22(2.637) \mathrm{mm}$, and the shortest distance was $0.00 \mathrm{~mm}$, whereas the longest distance was $11.00 \mathrm{~mm}$ (Table 1).

Based on the Spearman correlation test between pain rating scale and NVC distance to the REZ using 3D CISS MRI sequence, a weak correlation coefficient was observed between the shortest distance in NVC to REZ using the pain rating scale $(r=-0.39, p=0.021)$ (Figure 1a). The Spearman correlation coefficient between the NVC distance and the REZ on the lateral side using the pain rating scale demonstrated a moderate correlation coefficient $(r=-0.57, p=0.294)$ (Figure $1 b)$. The pain rating scale inversely correlated with the shortest distance and the distance on the lateral side. Pain as a symptom resulting from TN can become worse when the distance between NVC and REZ becomes shorter in the trigeminal nerve. However, on the medial side, no significant correlation was detected in this study $(r=-0.57, p=0.294)$ (Figure 1c).

\section{DISCUSSION}

$\mathrm{TN}$ is a unilateral facial pain that occurs in the trigeminal nerve and is primarily caused by NVC. ${ }^{1}$ Pain as a symptom resulting from TN has been reported to vary and correlate with the site of NVC compression in the trigeminal nerve. For asymptomatic patients with TN, NVC was found on the distal side of REZ, whereas it was primarily found on the proximal side of REZ in symptomatic patients. ${ }^{6}$ The aim of this study was to analyze the correlation between the NVC distance and the REZ using 3D CISS MRI sequence with pain as a symptom of TN measured using the pain rating scale.

The mean age of the study subjects was 53 years, with the range being 19-74 years, and subjects were divided into two groups, 19-50 and 51-74 years. Based on the age groups, the latter group consisted of more subjects than the former. The majority of the subjects were females. Joffroy et $\mathrm{al}^{2}$ reported that the ratio between females and males among patients with TN was 2:1 in the 40-year-old group and the peak age of onset was 50-60 years. The higher prevalence of TN in older people was related to the vascular degeneration process, whereas in females, the higher prevalence was associated with the accumulation of atherosclerosis."

In this cross-sectional study, we conducted 3D CISS MRI examination without contrast medium. We used several slices of thicknesses ranging from 0.20 to $0.80 \mathrm{~mm}$ to reveal more details about NVC and found that the combination of $0.2,0.4$, and $0.6 \mathrm{~mm}$ of slice thickness would provide the best information compared with other slice thicknesses. The variations in slice thickness in imaging provide more sequence details, which can help determine the vascular origins of NVC, than in subjects with single-slice thickness. The advantages of 3D CISS MRI sequences included construction into 3D imaging, a thinner slice thickness, and rapid acquisition time. Furthermore, all the 3D CISS MRI sequences performed better in NVC evaluation because of the ability to differentiate between an artery and a nerve.'

Using the 3D CISS MRI examination, we found that SCA was the most common cause of NVC in $52.38 \%$ of right-sided TN (Figure $2 \mathrm{a}$ ) and also in $57.14 \%$ of left-sided TN (Figure $2 \mathrm{~b}$ ). Similar results 
a. The shortest distance between NVC and RSZ

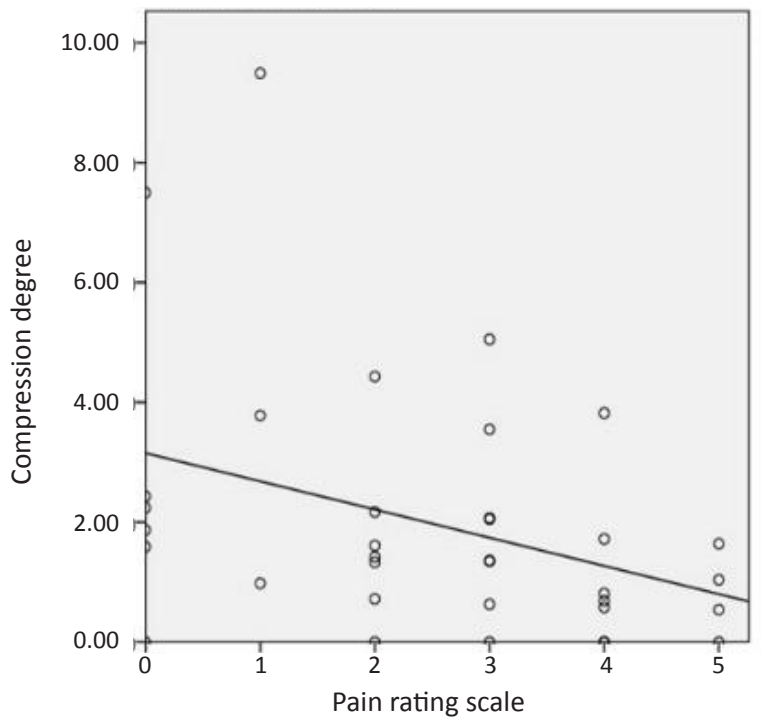

b. Lateral side

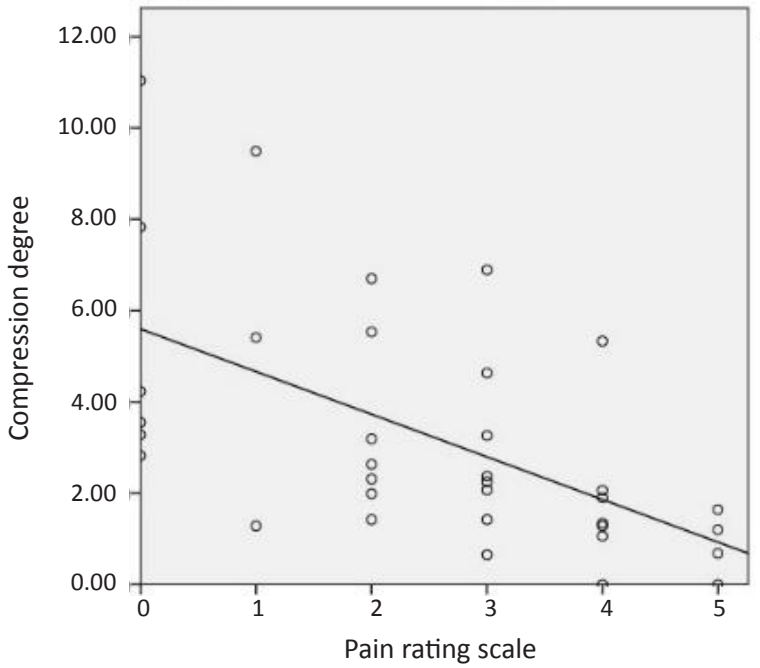

c. Medial side

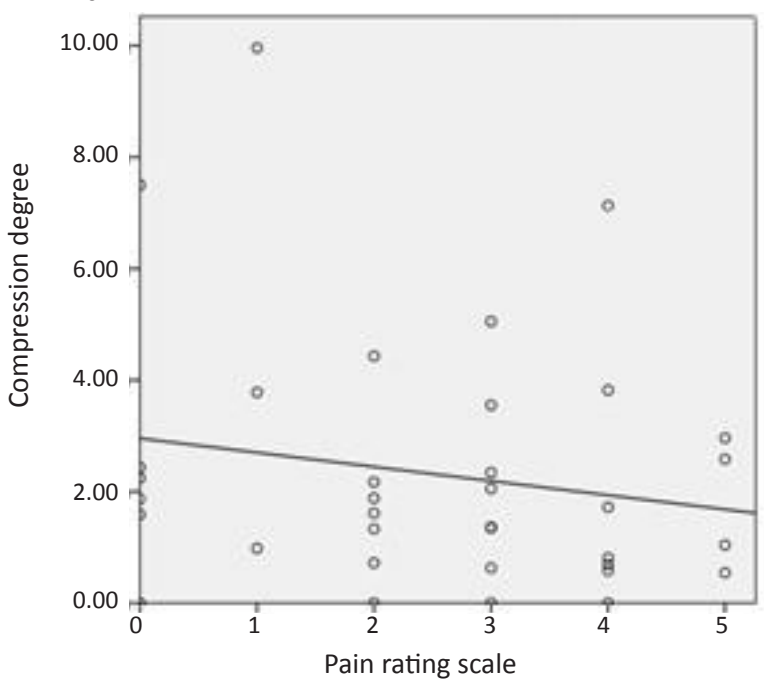

Figure 1. Correlation between pain assessment scale and the distance of NVC to REZ in patients with TN. (a) The shortest distance; (b) Lateral side; (c) Medial side. NVC=neurovascular compression; $\mathrm{REZ}=$ root exit zone; $\mathrm{TN}=$ trigeminal neuralgia were obtained by Cheng et $\mathrm{al}^{12}$ wherein SCA was the most common cause of compression, followed by the anteroinferior cerebellar artery (AICA) rather than other vessels in the pontine region such as the basilar artery and pontine veins. In this study, it was found that SCA originates from the proximal basilar artery, which is defined as typical configuration $1 .^{12}$ Krzyżewski et al' ${ }^{13}$ stated SCA generally originates bilaterally from the basilar artery as a single trunk and its diameter is significantly wider in that type compared with other anatomical variations.

The majority of the compressions in this study was found to be of third-grade, with the deviation sign image and a narrowing trigeminal nerve caliber. The etiology of third-grade compression was larger blood vessels such as the basilar and vertebral arteries rather than the SCA or AICA. The results of this study are not different from those of Blitz et $\mathrm{al}^{14}$ who evaluated TN using 3D CISS MRI sequences without contrast medium and found higher grading compression (grades 1, 2, and 3) of neurovascular conflict in the symptomatic side of the trigeminal nerve than that in the group of the asymptomatic side that had null grade. The neurovascular conflict in NVC is sufficient but not specific for causing symptoms.

The mean (SD) measurement of the shortest distance between NVC and REZ in this study was 2.1 (2.1) $\mathrm{mm}$. A closer distance between NVC and REZ could increase the pain. Some of the studies have evaluated the relationship between the severity of NVC and symptomatic and asymptomatic subjects. According to Suzuki et al, ${ }^{6}$ the mean distance was $0.94(1.27) \mathrm{mm}$ in the symptomatic nerve group, whereas it was greater in the asymptomatic nerve group (3.85 [2.69] mm). Lorenzoni et al' ${ }^{15}$ evaluated the distance between the NVC and the brainstem surface in symptomatic subjects and found that it was $<3 \mathrm{~mm}$ in 39 cases and $\geq 3 \mathrm{~mm}$ in 42 cases. However, they did not evaluate the distance in asymptomatic subjects.

In this study, there was an inverse correlation between pain assessedusing NRS and the NVC distance to the REZ $(r=-0.39, p=0.021)$. A closer distance between them could increase pain as a symptom of TN. Similarly, this study also demonstrated a correlation between NRS and compression distance on the lateral side $(r=-0.57, p<0.001)$. However, analysis on the medial side showed no correlation between the distance and the NRS because the mean 

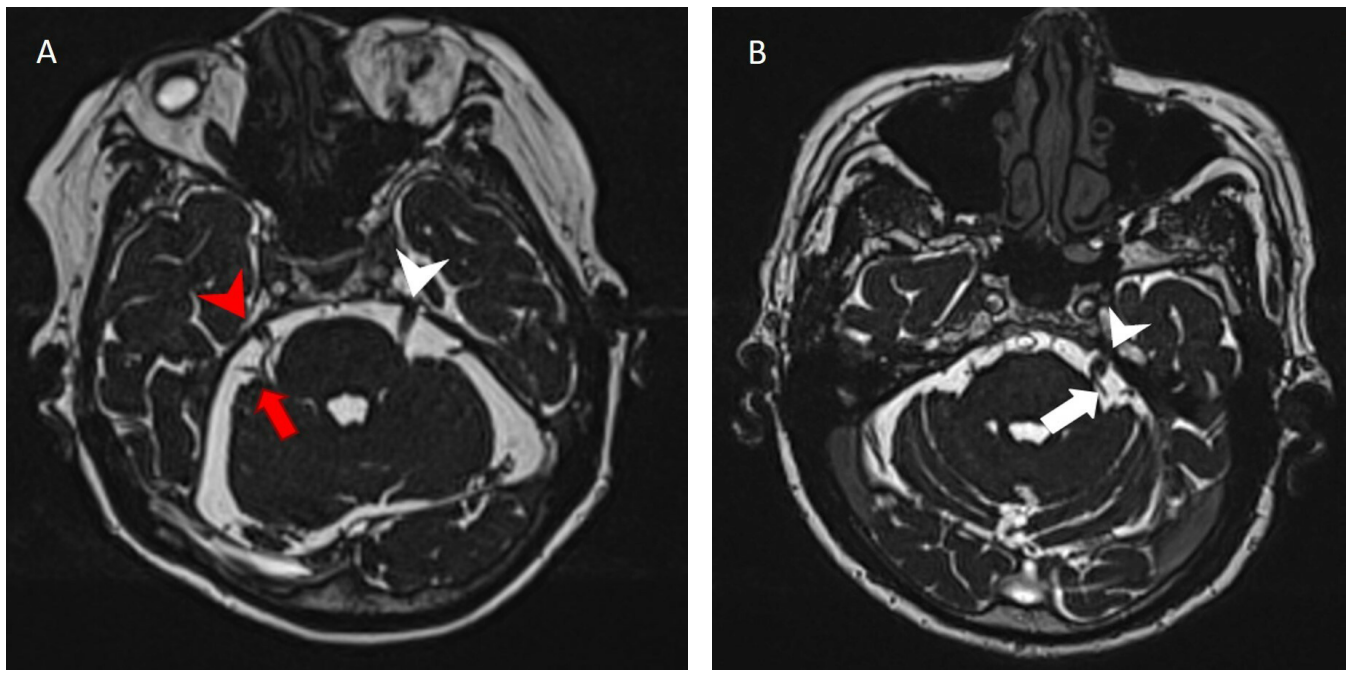

Figure 2. NVC vascular origin: (a) Female, 69 years old, with TN, both left TN (white arrowhead) and right TN (red arrowhead) had normal size and morphology. No evidence of thickening but right SCA (red arrow) ran through and compressed right TN; (b) Male, 63 years old, with TN. No evidence of thickening and dilatation of left TN (white arrowhead) and left superior cerebellar artery (white arrow) but left superior cerebellar artery (white arrow) compressed left TN (white arrowhead). NVC=neurovascular compression; TN=trigeminal neuralgia; SCA=superior cerebellar artery

distance was beyond the central myelin. Similar results were obtained by Suzuki et al, ${ }^{6}$ who reported that TN is primarily caused by NVC near to REZ. The REZ is the site where focal demyelination of the trigeminal nerve occurs due to constant compression by blood vessels suppressing the nerve. In this study, a lower distance between NVC and REZ correlates with a higher value in the pain rating scale.

This finding was supported by Tomii et $\mathrm{al}^{16}$ who found that the central myelin consisted of oligodendrocytes whose amount decreased more as they extended into the peripheral before being replaced by Schwann cells. Demyelination in the central myelin would cause severe trigeminal pain, especially in the proximal REZ. The central myelin on the medial side was $0.1-$ to $2.5-\mathrm{mm}$ long and $1.13-\mathrm{mm}$ long for the mean distance. However, on the lateral side, it was 0.17 - to $6.75-\mathrm{mm}$ long and the mean distance was $2.12 \mathrm{~mm}$. The mean distance in this study was $2.10 \mathrm{~mm}$, and the compression distance on the lateral side was $3.20 \mathrm{~mm} .^{7}$ Based on the findings from the literature review, the acquired mean distance fell under the area of central myelin, which would have correlated with NRS scores.

Therefore, this study has demonstrated that a closer distance between the NVC location and the REZ in the trigeminal nerve can increase pain as a manifestation of TN. This finding was interesting in that the location of NVC could result in variations in pain in patients with TN. Therefore, we expect that this study can provide more information about this topic and the 3D CISS MRI examination can be used as a protocol to evaluate trigeminal nerve and NVC contact.

There are some limitations exist in this study. First, due to the small number of patients with TN, the use of analgesics in the subjects as an exclusion criterion was not considered. The second limitation is the variation in slice thickness on 3D CSS MRI during the assessment of each subject as the usage of singleslice thickness would cause difficulties in determining the vascular origin and vascular compression location. The third limitation is the limited data to confirm the etiology and the vascular compression characteristics as only three patients underwent microvascular decompression surgery.

\section{Conclusions}

PAS evaluated using an NRS instrument showed an inverse correlation with the distance of NVC to the REZ of the trigeminal nerve. A closer distance between NVC and REZ of the trigeminal nerve could increase pain as a manifestation of TN.

\section{Conflict of Interest}

The authors affirm no conflict of interest in this study.

\section{Acknowledgment}

None.

Funding Sources

None. 


\section{REFERENCES}

1. Besta R, Shankar YU, Kumar A, Rajasekhar E, Prakash SB. MR 3D CISS-a novel imaging modality in diagnosing trigeminal neuralgia-a review. J Clin Diagn Res. 2016;10(3):ZE01-3.

2. Joffroy $A$, Levivier $M$, Massager $N$. Trigeminal neuralgia. Pathophysiology and treatment. Acta Neurol Belg. 2001;101(1):20-5.

3. National Initiative of Pain Control. Wong-Baker FACES Pain Rating Scale. Natl Initiat Pain Control. 2003;2-3.

4. $\quad$ Chen GQ, Wang XS, Wang L, Zheng JP. Arterial compression of nerve is the primary cause of trigeminal neuralgia. Neurol Sci. 2014;35(1):61-6.

5. Antonini G, Di Pasquale A, Cruccu G, Truini A, Morino S, Saltelli G, et al. Magnetic resonance imaging contribution for diagnosing symptomatic neurovascular contact in classical trigeminal neuralgia: a blinded case-control study and meta-analysis. Pain. 2014;155(8):1464-71.

6. Suzuki M, Yoshino N, Shimada M, Tetsumura A, Matsumura $\mathrm{T}$, Fukayama $\mathrm{H}$, et al. Trigeminal neuralgia: differences in magnetic resonance imaging characteristics of neurovascular compression between symptomatic and asymptomatic nerves. Oral Surg Oral Med Oral Pathol Oral Radiol. 2015;119(1):113-8.

7. Peker S, Kurtkaya O, Üzün I, Pamir MN. Microanatomy of the central myelin-peripheral myelin transition zone of the trigeminal nerve. Neurosurgery. 2006;59(2):354-9.

8. Gonçalves FG, do Amaral LL. Constructive interference in steady state imaging in the central nervous system. Eur Neurol Rev. 2011;6(2):138.
9. Tarnaris A, Renowden S, Coakham HB. A comparison of magnetic resonance angiography and constructive interference in steady state-three-dimensional Fourier transformation magnetic resonance imaging in patients with hemifacial spasm. Br J Neurosurg. 2007;21(4):375-81.

10. Jo KW, Kong DS, Hong KS, Lee JA, Park K. Long-term prognostic factors for microvascular decompression for trigeminal neuralgia. J Clin Neurosci. 2013;20(3):440-5.

11. Koh KK. Effects of estrogen on the vascular wall: vasomotor function and inflammation. Cardiovasc Res. 2002;55(4):714-26.

12. Cheng J, Meng J, Liu W, Zhang H, Hui X, Lei D. Nerve atrophy in trigeminal neuralgia due to neurovascular compression and its association with surgical outcomes after microvascular decompression. Acta Neurochir. 2017;159(9):1699-705.

13. Krzyżewski RM, Stachura MK, Stachura AM, Rybus J, Tomaszewski KA, Klimek-Piotrowska W, et al. Variations and morphometric analysis of the proximal segment of the superior cerebellar artery. Neurol Neurochir Pol. 2014;48(4):229-35.

14. Blitz AM, Northcutt B, Shin J, Aygun N, Herzka DA, Theodros $D$, et al. Contrast-enhanced CISS imaging for evaluation of neurovascular compression in trigeminal neuralgia: improved correlation with symptoms and prediction of surgical outcomes. Am J Neuroradiol. 2018;39(9):1724-32.

15. Lorenzoni J, David P, Levivier M. Patterns of neurovascular compression in patients with classic trigeminal neuralgia: a highresolution MRI-based study. Eur J Radiol. 2012;81(8):1851-7.

16. Tomii M, Onoue H, Yasue M, Tokudome S, Abe T. Microscopic measurement of the facial nerve root exit zone from central glial myelin to peripheral Schwann cell myelin. J Neurosurg. 2003;99(1):121-4. 